\title{
Spin-dependent analysis of two-dimensional electron liquids
}

\author{
C. Bulutay and B. Tanatar \\ Department of Physics, Bilkent University, 06533 Bilkent, Ankara, Turkey
}

(Received 22 September 2001; published 2 May 2002)

\begin{abstract}
Two-dimensional electron liquid (2D EL) at full Fermi degeneracy is revisited, giving special attention to the spin-polarization effects. First, we extend the recently proposed classical-map hypernetted-chain (CHNC) technique to the 2D EL, while preserving the simplicity of the original proposal. An efficient implementation of CHNC is given utilizing Lado's quadrature expressions for the isotropic Fourier transforms. Our results indicate that the paramagnetic phase stays to be the ground state until the Wigner crystallization density, even though the energy separation with the ferromagnetic and other partially polarized states become minute. We analyze compressibility and spin stiffness variations with respect to density and spin polarization, the latter being overlooked until now. Spin-dependent static structure factor and pair-distribution functions are computed; agreement with the available quantum Monte Carlo data persists even in the strong-coupling regime of the 2D EL.
\end{abstract}

DOI: $10.1103 / P h y s R e v B .65 .195116$

PACS number(s): 71.10.Ca, 75.10.Lp, 75.30.Kz

\section{INTRODUCTION}

A growing number of experimental reports making spin a tangible quantity, in particular, injection of sizeable percentage of spin-polarized carriers to semiconductors, ${ }^{1}$ surged a new wave of research efforts. Accordingly, the spin has become the central entity in the recently flourishing field of spintronics. ${ }^{2}$ On the technological side, much longer spinrelaxation time as compared to energy or momentum of a carrier, suggests information to be transmitted and processed utilizing the spin degrees of freedom. Whereas on the basic science side, the emerging possibility is that spin can play a nontrivial role even in the "nonmagnetic" phenomena.

Meanwhile, two-dimensional (2D) electronic systems have been of considerable interest because of technological relevance to high-mobility transistor geometry and because of novel physics brought by the enhanced role of many-body effects in lower dimensions. A current example is the recent interest in the experimentally observed metal-insulator transition in Si MOSFETs at very low temperatures. ${ }^{3}$ The spin polarization of the two phases is believed to help uncover the responsible mechanism. ${ }^{4,5}$ Historically, the relevant ground state of the 2D electronic systems has attracted theoretical attention through the idealized model of the electron liquid (EL). In this model, positive ionic lattice is smeared out into an inert background, preserving the overall charge neutrality. The quantum many-body system is formed by electrons representing the conduction electrons of a metal or a doped semiconductor. EL at zero temperature is characterized by two parameters $r_{s}$ and $\zeta$, describing inverse density and spin polarization. Over several decades polarization nature of the ground state of the 2D EL has been a debated issue. Within the Hartree-Fock approximation ground state becomes fully polarized (ferromagnetic) for $r_{s}>2$, whereas using the random-phase approximation (RPA) the transition point increases to 2.3 (Ref.6). A more refined approach including self-consistent local field corrections ${ }^{7}$ has determined a transition to the ferromagnetic state at $r_{s}=5.5$. In the lack of direct experimental verification, quantum Monte Carlo (QMC) simulations are believed to produce the most reliable results. Variational QMC simulations by $\mathrm{Ceperley}^{8}$ indicated the ferromagnetic phase to be stable above $r_{s} \simeq 13$. Later on Tanatar and Ceperley ${ }^{9}$ using more accurate fixed-node diffusion Monte Carlo simulations found the unpolarized (paramagnetic) phase to be the ground state till Wigner crystallization that is predicted to occur at $r_{s} \simeq 37$. In contrast, Rapisarda and Senatore ${ }^{10}$ again by means of diffusion Monte Carlo simulations found a first-order phase transition from the unpolarized to the fully polarized phase at $r_{s}=20$, and very recently upon including the backflow corrections the transition point has moved to $r_{s}=30$, quite close to Wigner crystallization density. ${ }^{11}$ An earlier work that included the backflow correlations did not find such a transition. ${ }^{12}$

For homogeneous classical fluids interacting through effective two-body forces, a technique known as hypernettedchain (HNC) approximation has been widely used. A set of coupled integral equations related to the pair-distribution function forms the basis of the HNC framework. ${ }^{13}$ Over the previous decades several variants of HNC have been introduced to deal with quantum liquids, such as the EL. In particular, the Fermi hypernetted-chain method provides a systematic way to improve the ground-state wave function while summing the bridge diagrams in classical statistical mechanics, a formidable task. ${ }^{14}$ Along this line simplifications were offered, such as the Jastrow variational HNC for dealing with the EL problem. ${ }^{15}$ More recently another formulation was proposed resulting in a single zero-energy Schrödinger-like equation for the pair-distribution function. ${ }^{16}$ Quite recently, Dharma-wardana and Perrot (DwP) suggested to examine quantum liquids again through a similar $\mathrm{HNC}$ framework. ${ }^{17}$ They envisioned this as a mapping of the quantum many-body system at zero temperature, to the $\mathrm{CF}$ at a particular temperature (the so-called quantum temperature) $T_{q}$, such that when the pair-distribution functions computed via $\mathrm{HNC}$ integral equations for the $\mathrm{CF}$ were used for the $\mathrm{EL}$ at zero temperature yield the correct correlation energy at that density. Availability of several QMC data, as mentioned above, for the unpolarized and fully polarized EL renders the extraction of $T_{q}$ possible. DwP's basic conjecture is that with $T_{q}$ determined as such, more exotic cases like the partially 
polarized and finite temperature EL will be readily accessible through the same HNC machinery. In a subsequent paper considering 3D EL, they have reported about the finite temperature case with applications to Kohn-Sham calculations. ${ }^{18}$ It needs to be mentioned that a theoretical proof is currently lacking for such a temperature mapping of a quantum system to a classical one, and the consequences must also be critically examined.

In this paper, we extend the classical-map HNC (CHNC) approach of DwP to the 2D EL at zero temperature to examine the nature of ground state. The finite temperature calculations for 2D EL including higher-order correlations (bridge terms) have recently been performed. ${ }^{19}$ In our calculation the bridge terms are not included to provide a quantitative assessment of their importance. We find that in the absence of bridge corrections the $2 \mathrm{D}$ electron system remains to be in the paramagnetic fluid phase. Based on the results of groundstate energies in the spin-polarized and unpolarized states we also calculate the compressibility and spin susceptibility of the 2D EL.

The paper is organized as follows. In the following section, we first describe our procedure for the extraction of $T_{q}$, and outline the CHNC technique for the 2D EL. Section III presents our results and comparison to QMC data whenever possible. Our conclusion and discussions are given in Sec. IV followed by the Appendix discussing an efficient implementation of the CHNC technique.

\section{THEORY}

We consider a partially polarized 2D EL at full Fermi degeneracy (i.e., zero temperature) having areal electronic densities $n_{1}\left(n_{2}\right)$ for the majority (minority) spins (i.e., $n_{1}$ $\left.\geqslant n_{2}\right)$, with the total density $n=n_{1}+n_{2}$, and $\zeta \equiv\left(n_{1}\right.$ $\left.-n_{2}\right) / n$; the coupling parameter of the many-body system is given by $r_{s}=1 / \sqrt{\pi n a_{B}^{* 2}}$. Here $a_{B}^{*}=\epsilon / m^{*} e^{2}$ is the effective Bohr radius (we take $\hbar=k_{B}=1$ ). The associated correlation energy (per particle) in 3D effective Rydberg units $\left(R^{*}\right.$ $\left.=e^{2} / 2 \epsilon a_{B}^{*}\right)$ is given by

$$
\begin{aligned}
E_{c}\left(r_{s}, \zeta\right)= & \frac{4 \sqrt{2}}{3 \pi r_{s}}\left[(1+\zeta)^{3 / 2}+(1-\zeta)^{3 / 2}\right] \\
& -\frac{2 \sqrt{2}}{r_{s}^{2}} \int_{0}^{r_{s}} d r_{s}^{\prime} \gamma\left(r_{s}^{\prime}\right)
\end{aligned}
$$

The first term on right-hand side corresponds to the negative of the exchange energy and $\gamma$ is defined as

$$
\gamma=\frac{1}{2} \int_{0}^{\infty} d q_{n}\left[1-S\left(q_{n}\right)\right],
$$

where $S(\cdot)$ is the static structure factor and $q_{n}$ is the wave number normalized to unpolarized Fermi wave number, $k_{F U}=\sqrt{2 \pi n}$. The coupling-constant integration in Eq. (1) requires $\gamma$ for a range of $r_{s}$ values. This is not very desirable for our fitting procedure to extract the quantum temperature $T_{q}$. Rather a local (in $r_{s}$ ) expression can be obtained by differentiation, yielding

$$
\gamma\left(r_{s}\right)=\frac{2}{3 \pi}\left[(1+\zeta)^{3 / 2}+(1-\zeta)^{3 / 2}\right]-\frac{1}{2 \sqrt{2}} \frac{d}{d r_{s}}\left[r_{s}^{2} E_{c}\left(r_{s}\right)\right]
$$

Functional forms for $E_{c}$ has been obtained in a number of QMC simulations. ${ }^{8-10}$ We extract $T_{q}$ by fitting over a broad range of $r_{s}$ values (from $r_{s}=0.25$ to 40 ) to the unpolarized $E_{c}$ expression proposed by Rapisarda and Senatore ${ }^{10}$ counting on its acclaimed accuracy. The result can be represented by a functional form

$$
\frac{T_{q}}{E_{F}}=\frac{1+a r_{s}}{b+c r_{s}},
$$

with $a=1.470342, b=6.099404$, and $c=0.476465$. A similar expression was obtained by DwP for the 3D EL. However, we stress that this equation should not be read as a $T_{q}\left(r_{s}\right)$ relation. Especially, when it comes to the partially polarized EL two different Fermi levels exist: $E_{F 1}$ and $E_{F 2}$ for the two spin populations. Therefore, we propose to extend the above expression by introducing a $\zeta$-weighted Fermi level as $\left\langle E_{F}\right\rangle \equiv x_{1} E_{F 1}+x_{2} E_{F 2}$, where $x_{s} \equiv n_{s} / n$, so that we use

$$
\frac{T_{q}}{\left\langle E_{F}\right\rangle}=\frac{1+a r_{s}}{b+c r_{s}}
$$

with the same numerical values for $a, b$, and $c$.

The spin-resolved pair-distribution function between spins $i$ and $j$ is given within the HNC framework as

$$
g_{i j}(\rho)=\exp \left[-\beta \phi_{i j}(\rho)+h_{i j}(\rho)-c_{i j}(\rho)\right],
$$

where $\beta=1 / T_{q},{ }^{20} h_{i j}(\rho)=g_{i j}(\rho)-1$, and $c_{i j}$ is the direct correlation function. Note that this HNC form for $g_{i j}$ assures its positiveness at any coupling strength, a condition severely violated by most other techniques. ${ }^{6,7}$ In Eq. (5) $\phi_{i j}$ is the pair potential between the spin species $i$ and $j$. Following DwP's approach for the 3D EL,

$$
\phi_{i j}(\rho)=\mathcal{P}(\rho) \delta_{i j}+V_{C o u}(\rho),
$$

where $\quad V_{C o u}(\rho)=\left(e^{2} / \epsilon \rho\right)\left[1-\exp \left(-\rho / \lambda_{t h}\right)\right]$ with $\lambda_{t h}$ $=\sqrt{\hbar^{2} \beta / \pi m^{*}}$; hence this is the Coulomb potential including the additional thermal diffraction correction, ${ }^{21}$ which ensures the correct behavior of $g_{12}(\rho \rightarrow 0) .{ }^{17} \mathcal{P}(\rho)$ is the so-called Pauli potential accounting for the exchange interaction between like spins, which is extracted from the known ${ }^{22}$ noninteracting [i.e., $V_{\mathrm{Cou}}(\rho) \equiv 0$ ] case (designated by the superscript 0 below)

$$
\beta \mathcal{P}(\rho)=-\ln \left[g_{i i}^{0}(\rho)\right]+h_{i i}^{0}(\rho)-c_{i i}^{0}(\rho) .
$$

We compare in Fig. 1 the Pauli potentials in 3D and 2D displaying the long-range behavior in the latter case.

Another set of equations follow from the OrnsteinZernike relation, which for a homogeneous system is utilized after transforming to wave number $q$ space as

$$
H_{i j}(q)=C_{i j}(q)+\sum_{s=1,2} x_{s} H_{i s}(q) C_{s j}(q),
$$




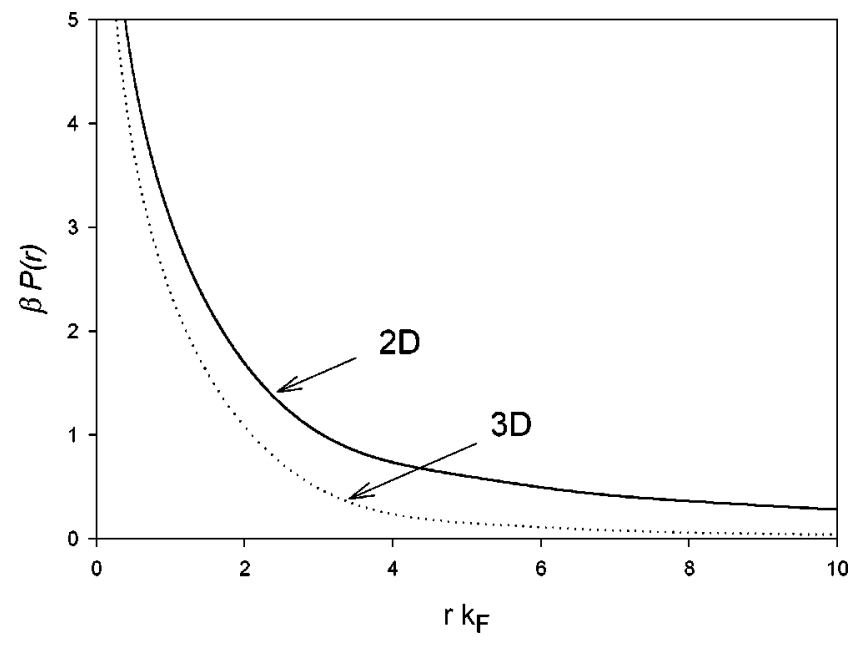

FIG. 1. Pauli potentials for the 2D and 3D electron liquids.

where we use the Fourier transform $H_{i j}(q)$ $\equiv n \int h_{i j}(\rho) e^{i \mathbf{q} \cdot \boldsymbol{\rho}} d \boldsymbol{\rho}$, and similarly for the other quantities. We solve these two sets until self-consistency is achieved (see the Appendix for details of the implementation).

Spin-resolved static structure factors are determined via

$$
S_{i j}(q)-\delta_{i j}=\sqrt{n_{i} n_{j}} \int d \boldsymbol{\rho}\left[g_{i j}(\rho)-1\right] e^{i \mathbf{q} \cdot \boldsymbol{\rho}} .
$$

For a chosen average electron, the probability of finding another electron (for either spin projection) at a distance $\rho$ away is given by the spin-averaged pair-distribution function, $g(\rho)$ as

$$
\begin{aligned}
g(\rho)= & \frac{1}{4}\left[(1+\zeta)^{2} g_{11}(\rho)+2\left(1-\zeta^{2}\right) g_{12}(\rho)\right. \\
& \left.+(1-\zeta)^{2} g_{22}(\rho)\right] ;
\end{aligned}
$$

its Fourier transform gives the spin-averaged static structure factor, $S(q)-1=n \int d \boldsymbol{\rho}[g(\rho)-1] e^{i \mathbf{q} \cdot \boldsymbol{\rho}}$ whose integral over $q$ relates to $\gamma\left(r_{s}\right)$ used in the correlation energy. The groundstate energy per particle (in $R^{*}$ ) is given as

$$
E\left(r_{s}, \zeta\right)=\frac{1+\zeta^{2}}{r_{s}^{2}}-\frac{4 \sqrt{2}}{3 \pi r_{s}}\left[(1+\zeta)^{3 / 2}+(1-\zeta)^{3 / 2}\right]+E_{c}
$$

Thermodynamic compressibility $(\kappa)$ and the static spin susceptibility $\left(\chi_{s}\right)$ are obtained by density $\left(r_{s}\right)$ and magnetization $(\zeta)$ derivatives of the energy resulting in

$$
\begin{aligned}
\frac{\kappa_{0}}{\kappa}=1 & -\frac{r_{s}}{\sqrt{2} \pi\left(1+\zeta^{2}\right)}\left[(1+\zeta)^{3 / 2}+(1-\zeta)^{3 / 2}\right] \\
& +\frac{r_{s}^{4}}{8\left(1+\zeta^{2}\right)}\left[\frac{\partial^{2} E_{c}}{\partial r_{s}^{2}}-\frac{1}{r_{s}} \frac{\partial E_{c}}{\partial r_{s}}\right], \\
\frac{\chi_{P}}{\chi_{s}}=1- & \frac{r_{s}}{\sqrt{2} \pi}\left[(1+\zeta)^{-1 / 2}+(1-\zeta)^{-1 / 2}\right]+\frac{r_{s}^{2}}{2} \alpha_{c},
\end{aligned}
$$

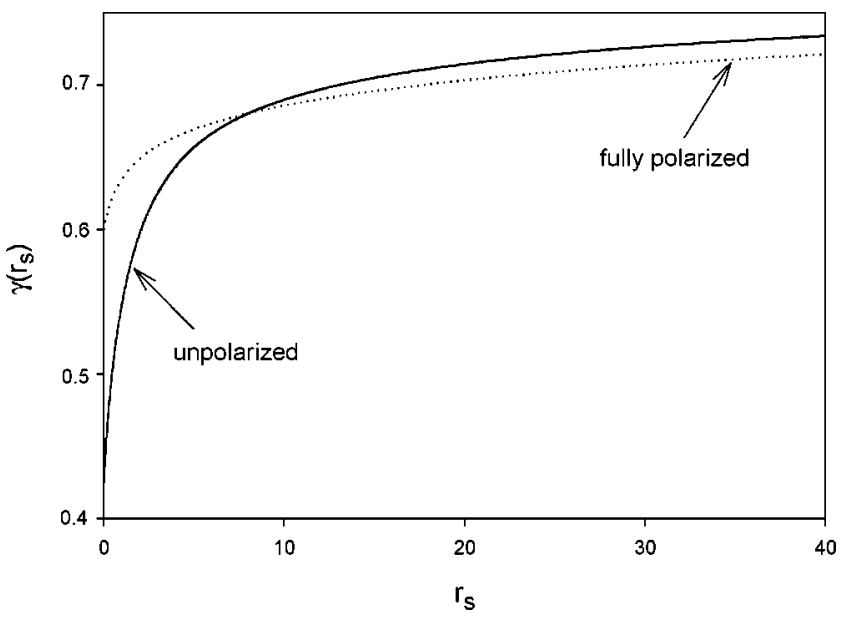

FIG. 2. $\gamma\left(r_{s}\right)$ for the unpolarized and fully polarized phases.

where $\kappa_{0}=\pi r_{s}^{4} / 2\left(1+\zeta^{2}\right)$ in $a_{B}^{* 2} / R^{*}$ units and $\chi_{P}$ $=m^{*} g^{2} \mu_{B}^{2} / 4 \pi \hbar^{2}$ are the corresponding quantities for the 2D ideal (noninteracting) Fermi gas at the same $r_{s}$ and $\zeta$ values; $\alpha_{c}=\partial^{2} E_{c} /\left.\partial \zeta^{2}\right|_{r_{s}}$ is the spin stiffness that contains effects beyond the Hartree-Fock approximation.

\section{RESULTS}

As it forms the core of our $T_{q}$ extraction procedure, in Fig. 2 we plot $\gamma\left(r_{s}\right)$ as defined by Eq. (3) for $\zeta=0,1$ values. Since the energies are calculated using $\gamma\left(r_{s}\right)$ very high accuracy is needed. In Fig. 3(a) we show CHNC pairdistribution function of the unpolarized phase at $r_{s}=1,5,10$, and 20, and compare with the tabulated QMC results. ${ }^{9}$ Again considering the unpolarized phase, the spin-dependent components, $g_{i j}$, are shown in Fig. 3(b) at $r_{s}=40$ together with the QMC fluid phase results of Rapisarda and Senatore. ${ }^{10}$ Figure 4 illustrates the the family of curves for the pairdistribution function at $r_{s}=1$ obtained by varying $\zeta$ from 0 to 1 . These results establish the overall reliability of the CHNC method.

The contact value of the paramagnetic pair-distribution function, i.e., $g(\rho=0) \equiv \frac{1}{2} g_{12}(0)$ is also of special importance. ${ }^{18}$ Very recently a model expression for $g(0)$ was offered $^{23}$ interpolating between the high-density and close to Wigner crystallization regimes, expressed as

$$
g(0)=\frac{1 / 2}{1+1.372 r_{s}+0.083 r_{s}^{2}}
$$

In Fig. 5 we compare this expression with that extracted from CHNC. Agreement is seen to exist only in the highdensity region. The available QMC data further suggest that the interpolation given by Eq. (14) overestimates the contact value for the low-density regime.

One can also calculate the spin-resolved static structure factors [See Eq. (9)]. Choosing $r_{s}=10$ case for illustration purposes, Fig. 6 displays the unpolarized and fully polarized phases, again comparing with the tabulated QMC data. ${ }^{9}$ The 

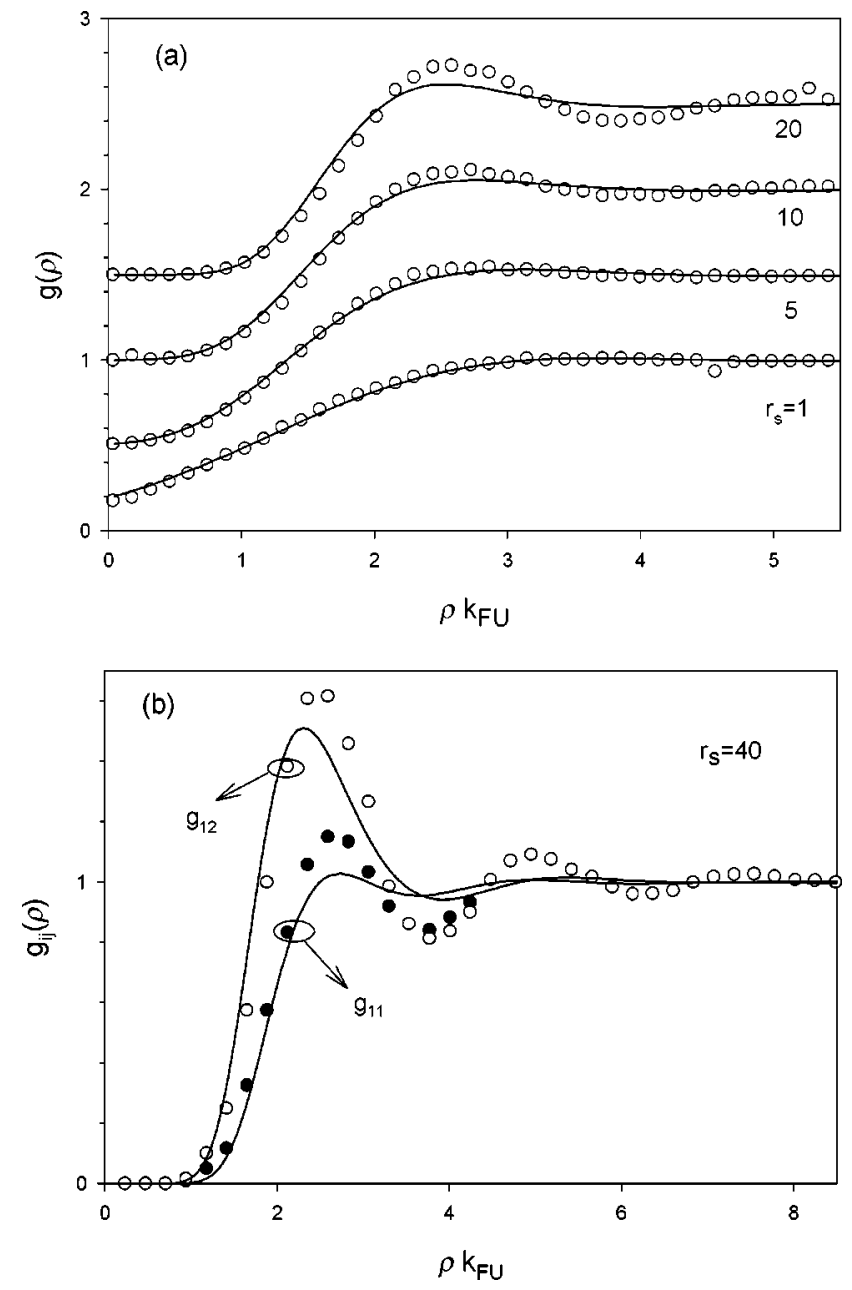

FIG. 3. Unpolarized phase pair-distribution functions: (a) spinaveraged $g(\rho)$ for $r_{s}=1,5,10$, and 20, comparing CHNC (solid lines) and Tanatar and Ceperley's QMC results (circles); (b) spindependent $g_{i j}(\rho)$ at $r_{s}=40$, comparing CHNC (solid lines) and Rapisarda and Senatore's QMC results (circles). Curves in (a) are successively vertically displaced by 0.5 unit for clarity; QMC data in (b) is based on our graphical readings from Ref. 10. $k_{F U}$ $=\sqrt{2 \pi n}$ is the unpolarized Fermi wave number.

shift in the peaks of $S(q)$ mainly results from using the unpolarized Fermi wave number $k_{F U}$ normalization also for the fully spin-polarized case.

As mentioned above, variation of the total energy of the 2D EL with respect to density and spin polarization has been particularly needed in addressing the debated nature of its ground state. Rapisarda and Senatore ${ }^{10}$ and more recently Varsano and co-workers ${ }^{11}$ have reported the ferromagnetic phase to be the ground state towards the Wigner crystallization densities. Even though we have fitted to their unpolarized correlation energy ${ }^{10}$ while extracting the quantum temperature $T_{q}$, CHNC results shown in Fig. 7 indicate the unpolarized phase to be the ground state well up to the Wigner density, in agreement with the QMC results of Tanatar and Ceperley ${ }^{9}$ and Kwon. ${ }^{12}$ However, the energy difference between the paramagnetic and ferromagnetic phases approaches $1 \mathrm{mRy}$ (see, Fig. 7 inset), and such an accuracy of the CHNC results can be questionable, especially with the

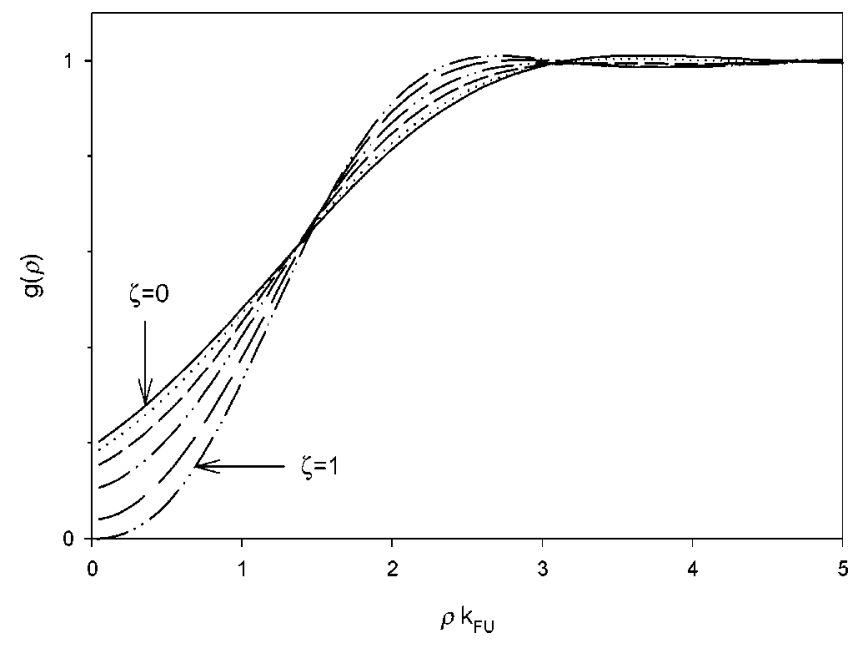

FIG. 4. Family of pair-distribution functions at $r_{s}=1$ for $\zeta=0$, $0.3,0.5,0.7,0.9$, and 1 (respectively from top to bottom on the left-hand side of the figure). $k_{F U}=\sqrt{2 \pi n}$ is the unpolarized Fermi wave number.

backflow corrections and three-body and higher correlations $^{24,25}$ unaccounted in the CHNC case. Nevertheless, as compared to other techniques that predict a transition to ferromagnetic phase at higher densities, ${ }^{6,7} \mathrm{CHNC}$ provides a remarkable improvement.

Figure 8 shows the dependence of inverse compressibility on $r_{s}$ for the unpolarized and fully spin-polarized cases. The latter has lower compressibility predominantly following from the increased exchange pressure. The inert nature of the positive background of the EL model accounts only for the electronic contribution to compressibility; this is seen to become negative at $r_{s}=2.04$ and 3.07 for the unpolarized and fully polarized cases, respectively. Finally, in Fig. 9 we display the spin susceptibility of the unpolarized phase. The Hartree-Fock susceptibility is seen to reverse sign at $r_{s}=2$ in accordance with the associated phase transition to the ferromagnetic state. The $\mathrm{CHNC}$ result initially shows an enhance-

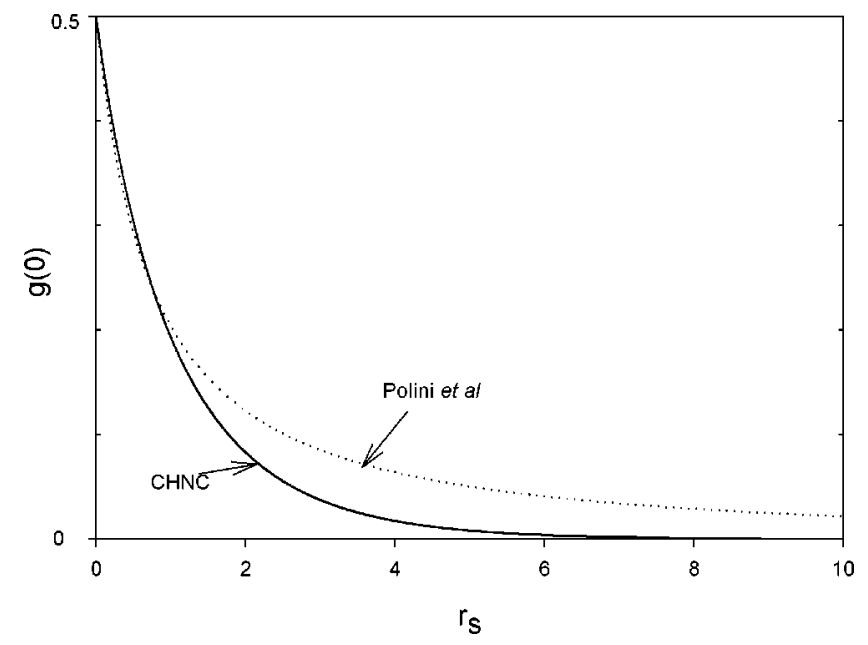

FIG. 5. Contact (i.e., $\rho=0$ ) value of the pair-distribution function of the unpolarized phase: $\mathrm{CHNC}$ versus model interpolation expression of Ref. 22. 


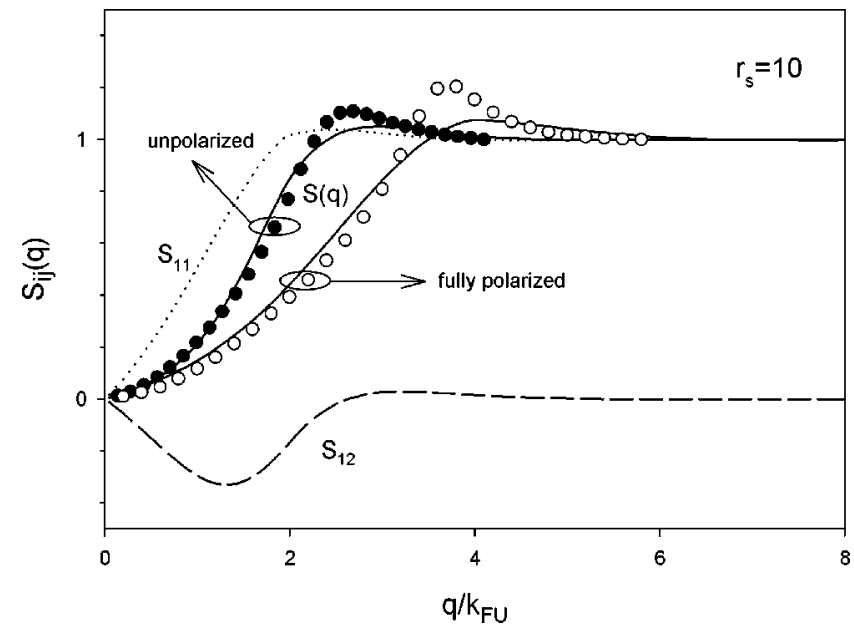

FIG. 6. Spin-dependent and spin-averaged static structure factors at $r_{s}=10$ for the unpolarized and fully polarized phases. Tanatar and Ceperley's QMC $S(q)$ data (circles) are also included for comparison. $k_{F U}=\sqrt{2 \pi n}$ is the unpolarized Fermi wave number.

ment over the Hartree-Fock result but then monotonically decreases, however, always remaining positive, in accord with our previous finding that the CHNC unpolarized phase is the ground state for all the densities considered (see Fig. 7). The inset in Fig. 9 shows behavior of the associated CHNC spin stiffness coefficient.

\section{CONCLUSION}

In this work we proposed an extension and efficient implementation of the $\mathrm{CHNC}$ technique to $2 \mathrm{D}$ while retaining its original simplicity. A recent calculation by $\mathrm{DwP}^{19}$ also discusses the the 2D and finite temperature extension of their $\mathrm{CHNC}$ technique. At variance with this work, for the parallel spin interactions they include the bridge term ${ }^{13}$ of the HNC technique. The agreement of our results (without the bridge correction) with the available QMC data is quite suggestive,

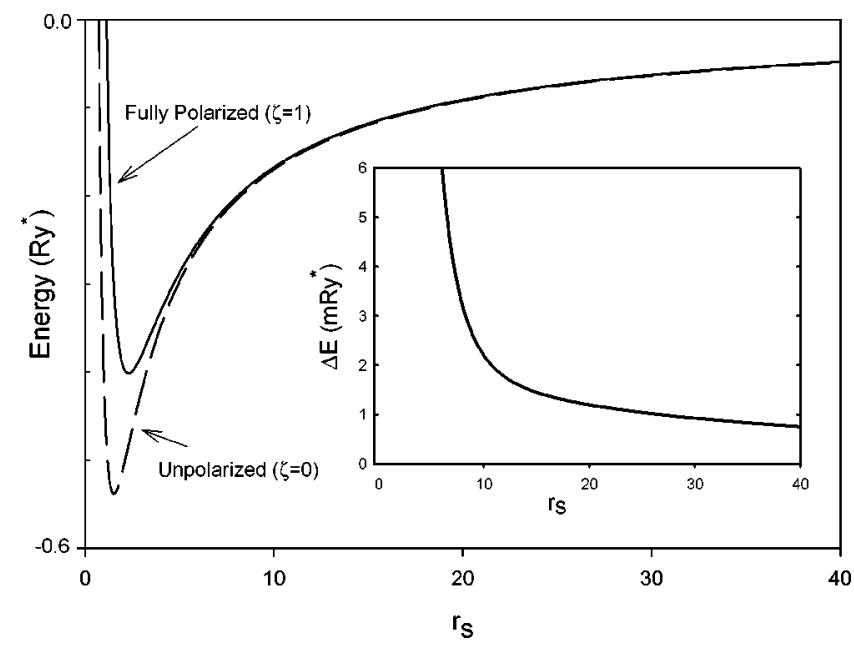

FIG. 7. Total energy of the unpolarized and fully polarized phases. Inset illustrates how much the fully polarized phase is higher in energy in milliRydbergs from the unpolarized phase.

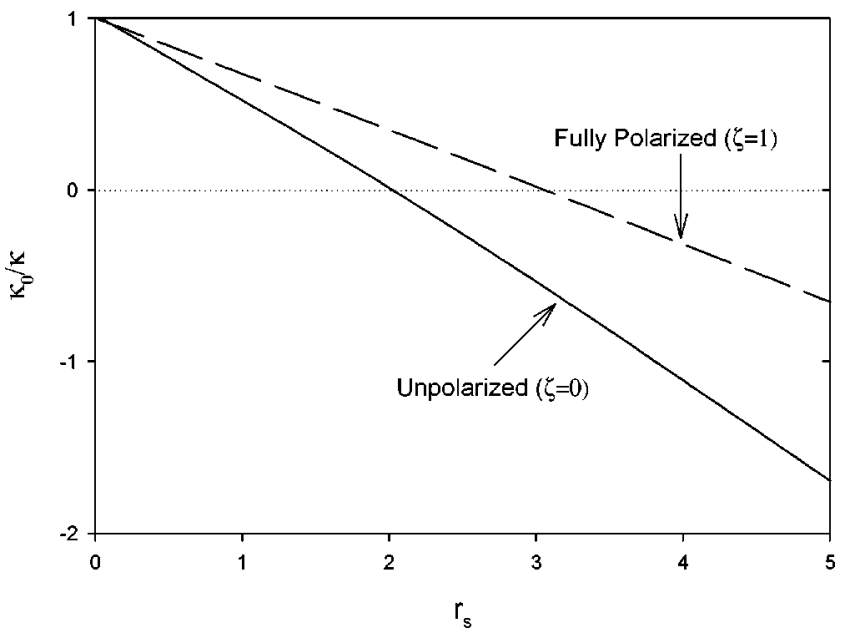

FIG. 8. Inverse compressibility normalized to $2 \mathrm{D}$ free fermion value $\left(\kappa_{0}\right)$ of the unpolarized and fully polarized phases.

given the fact that for more involved problems like doublelayer systems, inclusion of bridge terms becomes less straightforward. We have also analyzed the compressibility and spin susceptibility of the 2D EL.

The ground state of the 2D EL comes out to be the unpolarized phase, while the energy difference with the ferromagnetic phase diminishes to a milliRydberg value where such an accuracy of the CHNC results is moot. However, a similar concern can be addressed for the QMC simulations that are done for a finite number of particles $(N)$, with $N \sim 100$ and extrapolations to bulk limit $N \rightarrow \infty$ are obtained following some ansatz. ${ }^{9,10}$ Hence, decreasing the error bars becomes a daunting task, while still leaving doubts over the final results. Our preliminary assessment here suggests $\mathrm{CHNC}$ as a practical alternative for the QMC simulations, whereas other techniques fall far too short, producing negative pairdistribution functions and transitions to fully polarized phase at unrealistically high densities. Several issues on CHNC remain to be dealt in near future. Most important is a better

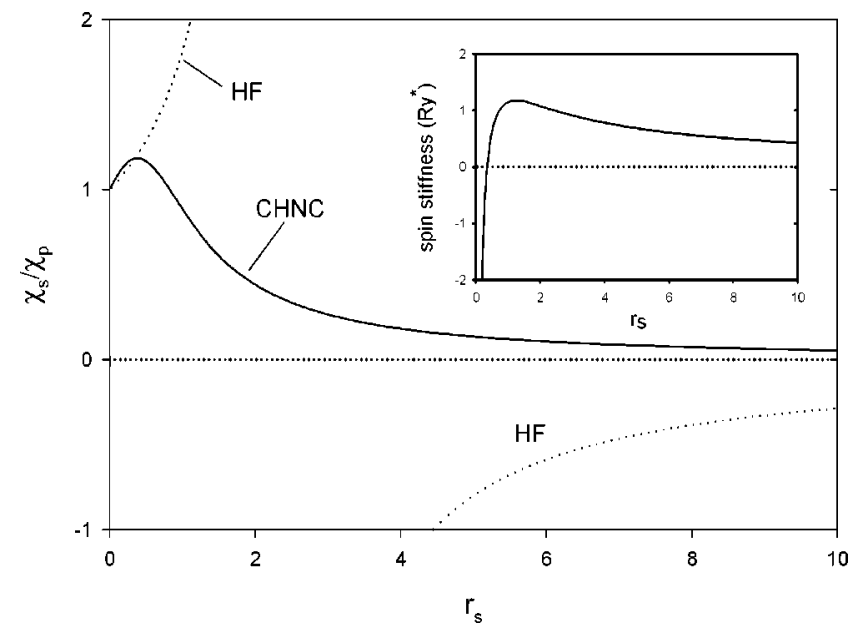

FIG. 9. Spin susceptibility normalized to 2D noninteracting Pauli value $\left(\chi_{P}\right)$ calculated via CHNC and Hartree-Fock methods. Inset shows the CHNC spin stiffness $\alpha_{c}$, in units of Rydberg. 
theoretical understanding for the temperature mapping of the quantum many-body system to a classical fluid. Similarly, a rigorous dielectric formalism for $\mathrm{CHNC}$ will be essential for analyzing collective excitations and also including effects of the disorder which generally lead to nontrivial outcomes. Finally, addressing finite-temperature effects is highly desirable which becomes fairly easy within the proposed CHNC framework.

\section{ACKNOWLEDGMENTS}

This work was supported by the Scientific and Technical Research Council of Turkey (TUBITAK), by NATO-SfP, the Turkish Department of Defense, and by the Turkish Academy of Sciences (TUBA).

\section{APPENDIX: SOME DETAILS ON THE IMPLEMENTATION}

The crux of the HNC framework is formed by Eqs. (5) and (8) in the coordinate ( $\boldsymbol{\rho})$ and wave vector (q) spaces to be solved self-consistently. From the computational perspective this necessitates an efficient implementation of the Fourier transform, the rest of the operations being solely algebraic. As we mention in the main text, for convenience we prefer to use Fourier transforms scaled by the total areal electronic density, so that

$$
\begin{gathered}
f(\rho)=\frac{1}{n} \int \frac{d^{2} q}{(2 \pi)^{2}} F(q) e^{-i \boldsymbol{\rho} \cdot \mathbf{q}}, \\
F(q)=n \int d^{2} \rho f(\rho) e^{i \boldsymbol{\rho} \cdot \mathbf{q}} .
\end{gathered}
$$

In the remaining part of this Appendix we shall use wave numbers (distances) normalized to $k_{F U}\left(1 / k_{F U}\right)$, the unpolarized Fermi wave number $\left(k_{F U}=\sqrt{2 \pi n}\right)$. For the Fourier transform of functions having no angular dependence (i.e., isotropic), Lado has offered simple quadrature expressions. ${ }^{26}$ The only approximation follows from setting $f\left(\rho_{\max }\right)$ $=F\left(Q_{\max }\right)=0$ at some presumably large $\rho_{\max }$ and $Q_{\max }$ values. For a total number of $N$ points - same in both spacesthe grid points are allocated at $\rho_{l}=\left(\mu_{l} / \mu_{N}\right) \rho_{\max }$ and $q_{m}$ $=\left(\mu_{m} / \rho_{\max }\right) \equiv\left(\mu_{m} / \mu_{N}\right) Q_{\max }$, where $\mu_{m}$ is the $m$ th positive root of the zeroth-order Bessel function $J_{0}(\cdot)$. Lado's quadrature expressions for the 2D Fourier transform pair then become

$$
\begin{gathered}
f\left(\rho_{l}\right)=\sum_{m=1}^{N-1} \Delta q_{m} q_{m} F\left(q_{m}\right) J_{0}\left(q_{m} \rho_{l}\right), \\
F\left(q_{m}\right)=\sum_{l=1}^{N-1} \Delta \rho_{l} \rho_{l} f\left(\rho_{l}\right) J_{0}\left(q_{m} \rho_{l}\right),
\end{gathered}
$$

where

$$
\begin{aligned}
\Delta \rho_{l} & \equiv \frac{2}{Q_{\text {max }}^{2} \rho_{l}\left[J_{1}\left(Q_{\text {max }} \rho_{l}\right)\right]^{2}}, \\
\Delta q_{m} & \equiv \frac{2}{\rho_{\text {max }}^{2} q_{m}\left[J_{1}\left(q_{m} \rho_{\text {max }}\right)\right]^{2}} .
\end{aligned}
$$

It needs to be mentioned that the Coulomb potential is long ranged and sudden truncation of the integrals at some $\rho_{\max }$ value results in rapid oscillations in its Fourier transform. We remedy this by first windowing it by a cosine square profile such that, $V_{\text {Cou }}(\rho) \rightarrow V_{\text {Cou }}(\rho) \times \cos ^{2}\left[\left(\pi / 2 \rho_{\max }\right) \rho\right]$. For the unpolarized case we typically use $N=800, Q_{\max } \simeq \rho_{\max }=50$. To assure that long-range tails are not affected by such a choice, we doubled the size of the window as $N=3200$, $Q_{\max } \simeq \rho_{\max }=100$, and found that the change in the results were indiscernible.
${ }^{1}$ M. Johnson and R. H. Silsbee, Phys. Rev. Lett. 55, 1790 (1985); R. Fiederling, M. Keim, G. Reuscher, W. Ossau, G. Schmidt, A. Waag, and L. Molenkamp, Nature (London) 402, 787 (1999); Y. Ohno, D. Young, B. Beschoten, F. Matsukura, H. Ohno, and D. Awschalom, ibid. 402, 790 (1999); B. T. Jonker, Y. D. Park, B. R. Bennett, H. D. Cheong, G. Kioseoglou, and A. Petrou, Phys. Rev. B 62, 8180 (2000); C. J. Hill, X. Cartoixà, R. A. Beach, D. L. Smith, and T. C. McGill, cond-mat/0010058 (unpublished).

${ }^{2}$ M. Johnson, IEEE Spectrum 37, 33 (2000); P. Ball, Nature (London) 404, 918 (2000); R. Matthews, New Scientist 157, 2123 (1998); D. D. Awschalom and J. M. Kikkawa, Phys. Today 52(6), 33 (1999); S. Das Sarma, J. Fabian, X. Hu, and I. Zutić, Superlattices Microstruct. 27, 290 (2000).

${ }^{3}$ For a review, see, E. Abrahams, S. V. Kravchenko, and M. P. Sarachik, Rev. Mod. Phys. 73, 251 (2001).

${ }^{4}$ T. Okamoto, K. Hosoya, S. Kawaji, and A. Yagi, Phys. Rev. Lett. 82, 3875 (1999).

${ }^{5}$ S. A. Vitkalov, H. Zheng, K. M. Mertes, M. P. Sarachik, and T. M. Klapwijk, Phys. Rev. Lett. 85, 2164 (2000).
${ }^{6}$ A. K. Rajagopal and J. C. Kimball, Phys. Rev. B 15, 2819 (1977).

${ }^{7}$ B. Davoudi and M. P. Tosi, cond-mat/0107519 (unpublished).

${ }^{8}$ D. Ceperley, Phys. Rev. B 18, 3126 (1978).

${ }^{9}$ B. Tanatar and D. M. Ceperley, Phys. Rev. B 39, 5005 (1989).

${ }^{10}$ F. Rapisarda and G. Senatore, Aust. J. Phys. 49, 161 (1996).

${ }^{11}$ D. Varsano, S. Moroni, and G. Senatore, Europhys. Lett. 53, 348 (2001).

${ }^{12}$ Y. Kwon, J. Korean Phys. Soc. 31, 862 (1997).

${ }^{13}$ J. P. Hansen and I. R. McDonald, Theory of Simple Liquids (Academic, New York, 1986).

${ }^{14}$ S. Fantoni and S. Rosati, Nuovo Cimento A 25, 593 (1975).

${ }^{15}$ J. G. Zabolitzky, Phys. Rev. B 22, 2353 (1980); C. E. Campbell and J. G. Zabolitzky, ibid. 27, 7772 (1983); L. J. Lantto, ibid. 22, 1380 (1980).

${ }^{16}$ A. Kallio and J. Piilo, Phys. Rev. Lett. 77, 4237 (1996).

${ }^{17}$ M. W. C. Charma-wardana and F. Perrot, Phys. Rev. Lett. 84, 959 (2000).

${ }^{18}$ F. Perrot and M. W. C. Dharma-wardana, Phys. Rev. B 62, 16536 (2000). 
${ }^{19}$ F. Perrot and M. W. C. Dharma-wardana, Phys. Rev. Lett. 87, 206404 (2001).

${ }^{20} \beta=1 / k_{B} \sqrt{T_{q}^{2}+T^{2}}$ for the case of a quantum system at a finite temperature $T>0$, however, throughout this work we deal with fully degenerate $\mathrm{EL}$ (i.e., $T \equiv 0$ ).

${ }^{21}$ H. Minoo, M. M. Gombert, and C. Deutsch, Phys. Rev. A 23, 924 (1981)

${ }^{22}$ At zero temperature for the noninteracting case, $g_{i i}^{0}\left(\rho k_{F i}\right)=1$ $-\left[2 J_{1}\left(\rho k_{F i}\right) / \rho k_{F i}\right]^{2}$ and $g_{12}^{0} \equiv 1$.

${ }^{23}$ M. Polini, G. Sica, B. Davoudi, and M. P. Tosi, J. Phys.: Condens. Matter 13, 3591 (2001).

${ }^{24}$ D. Pines and P. Nozières, The Theory of Quantum Liquids (Addison-Wesley, New York, 1989), Vol. I.

${ }^{25}$ Y. Kwon, D. M. Ceperley, and R. M. Martin, Phys. Rev. B 48, 12 037 (1993).

${ }^{26}$ F. Lado, J. Comput. Phys. 8, 417 (1971). 